\title{
Preterm birth and ventilation decrease surface density of glomerular capillaries in lambs, regardless of postnatal respiratory support mode
}

\author{
Eveline Staub ${ }^{1,2}$, Mar Janna Dahl ${ }^{2}$, Calan Yost ${ }^{2}$, Sydney Bowen², Toshio Aoki², Adam Blair ${ }^{2}$, Zhengming Wang ${ }^{2}$, Donald M. Null2,3, \\ Bradley A. Yoder ${ }^{2}$ and Kurt H. Albertine ${ }^{2}$
}

BACKGROUND: Prematurity is often complicated by respiratory support, including invasive mechanical ventilation (IMV) and noninvasive support (NIS). Compared with IMV, NIS reduces injury to the lung and brain. Prematurity may also disrupt glomerular architecture. Whether NIS differentially affects glomerular architecture is incompletely understood. We hypothesized that IMV would lead to greater disruption of glomerular architecture than NIS.

METHODS: This is a secondary analysis of kidneys from moderately preterm lambs delivered at $\sim 131 \mathrm{~d}$ gestation (term $\sim 150$ d) that had antenatal steroid exposure and surfactant treatment before resuscitation by IMV. At $\sim 3 \mathrm{~h}$ of age, half of the lambs were switched to NIS. Support was for $3 \mathrm{~d}$ or $21 \mathrm{~d}$. Structural indices of glomerular architecture were quantified.

RESULTS: The number of glomerular generations was unaffected by moderate preterm birth and respiratory support, either IMV or NIS. At $3 \mathrm{~d}$ and $21 \mathrm{~d}$ of IMV or NIS, glomerular capillary surface density was not different. Glomerular capillary surface density was significantly lower in the inner and outer cortex compared with unventilated gestation age-matched or postnatal age-matched reference lambs.

CONCLUSION: Moderate preterm birth and invasive or noninvasive respiratory support decreases glomerular capillarization in the lamb kidney. This adverse effect on glomerular development may contribute to increased risk for adult-onset hypertension and renal dysfunction.

A lthough the absolute number of nephrons appears to be in place by around $36 \mathrm{wk}$ gestation in the kidney of the human fetus, maturation of nephrons is only about $60 \%$ complete (1-4). After preterm birth, the immaturity of the kidneys makes them vulnerable to perinatal and postnatal stresses that may disrupt kidney development. Increasing evidence of prematurity as a risk factor for impaired renal function and hypertension with onset early in childhood $(5,6)$ suggests that altered postnatal renal maturation has a long-term impact on renal health.
Management of premature infants is often complicated by the need for either invasive or noninvasive respiratory support for respiratory distress (7). Invasive support entails endotracheal intubation with invasive mechanical ventilation (IMV), whereas noninvasive support (NIS) provides respiratory gases via the nose/pharynx of spontaneously breathing neonates. Acute lung injury during IMV in adults is associated with acute kidney injury, and various animal and human studies provide evidence of the interplay between IMV and renal function $(8,9)$. The effects of IMV on the immature kidney are incompletely understood. An autopsy study of premature human infants showed ongoing, but altered postnatal glomerulogenesis and glomerular maturation (10). However, the causes of illness and death varied, not all of the preterm infants had respiratory failure, and others had intrauterine growth restriction. Therefore, the impact of IMV per se could not be determined. Analysis of the kidney of premature baboons supported by IMV for up to $21 \mathrm{~d}$ revealed that glomerulogenesis continued postnatally, but postnatal glomerular development was disrupted (11). Both studies examined subjects born before the completion of glomerulogenesis and may demonstrate effects that are different in preterm subjects born around or after the time when all glomeruli are formed. Both studies also reveal that vulnerability of glomeruli is greater in the outer cortex than the inner cortex, a finding that matches the centrifugal wave of glomerulogenesis (12).

Noninvasive respiratory support (NIS) is widely used for preterm infants. Together with other measures such as antenatal steroid exposure, surfactant replacement therapy, and caffeine administration, NIS is associated with less bronchopulmonary dysplasia (BPD)compared with IMV (13). Evidence from preterm lambs and preterm baboons shows that NIS,compared with IMV, not only reduces injury to the lung, but also the brain (14-16). Whether IMV and NIS have differential effects on glomerular development is not known.

The aim of this study is to identify differential effects of IMV vs. NIS on glomerular architecture in the kidneys of preterm lambs. Our study is a secondary analysis of fixed kidneys from

'Division of Neonatology, University Children's Hospital Basel UKBB, Basel, Switzerland; '2Division of Neonatology, Department of Pediatrics, University of Utah, Salt Lake City, Utah; ${ }^{3}$ Division of Neonatology, University of California, Davis, California. Correspondence: Kurt H. Albertine (kurt.albertine@hsc.utah.edu)

Received 30 March 2016; accepted 4 November 2016; advance online publication 24 May 2017. doi:10.1038/pr.2017.1 
moderately preterm lambs $(\sim 131 \mathrm{~d}$ gestation; term $\sim 150 \mathrm{~d})$ that were supported by either IMV or NIS for $3 \mathrm{~d}$ or $21 \mathrm{~d}$ for the purpose of identifying effects of mode of respiratory support on alveolarization in the immature lung $(14,15)$. For this study, we quantified glomerular generations, glomerular capillary surface density $\left(\mathrm{SV}_{\mathrm{gc}}\right)$, and glomerular surface area. We hypothesized that IMV would lead to greater disruption of glomerular architecture than NIS. Our results suggest that glomerular development is vulnerable to disruption by either invasive or noninvasive respiratory support.

\section{METHODS}

The protocols adhered to the American Physiological Society/ National Institutes for Health guidelines for humane use of animals for research, and were approved by the Institutional Animal Care and Use committee at the University of Utah, Health Sciences Center.

Preterm Lamb Groups for Respiratory Support for $\mathbf{3}$ d or $\mathbf{2 1 ~ d ~}$ This is a secondary analysis of kidneys from moderately preterm lambs delivered at $\sim 131 \mathrm{~d}$ gestation that we studied for lung outcomes (15). At the time of the lung study, indices of renal function were not measured, other than urine output. The methods for chronically ventilated preterm lambs are reported $(14,15)$. Briefly, time-pregnant ewes at $-131 \mathrm{~d}$ of gestation (term $\sim 150 \mathrm{~d}$ ) were used. Glomerulogenesis finishes at $-120 \mathrm{~d}$ of gestation in sheep (17); therefore, our model of moderately preterm ventilated lambs examined glomerular development after glomeruli are formed. Preterm lambs were exposed to antenatal betamethasone $(6 \mathrm{mg} / \mathrm{kg}$; American Regent, Shirley, NY), perinatal surfactant (240 mg; Curosurf, Chiesi, Parma, Italy), and postnatal caffeine citrate (loading dose of $15 \mathrm{mg} /$ kg; Paddock Laboratories, Minneapolis, MN; $5 \mathrm{mg} / \mathrm{kg} / \mathrm{d}$ ). Before, delivery, fetuses were prospectively assigned to one of two modes of respiratory support: (i) invasive mechanical ventilation (IMV) or (ii) NIS. After operative delivery, preterm lambs were supported by IMV (Bird VIP ventilator, Palm Springs, CA), with warmed, humidified gases. Target respiratory gas parameters were partial pressure of arterial oxygen $\left(\mathrm{PaO}_{2}\right)$ of $60-80 \mathrm{mmHg}$, partial pressure of arterial carbon dioxide $\left(\mathrm{PaCO}_{2}\right)$ of $45-60 \mathrm{mmHg}$, and $\mathrm{pH}$ of 7.25-7.35.

Preterm lambs assigned to NIS were weaned from IMV at $2-3 \mathrm{~h}$ of postnatal life $(14,15)$. An uncuffed Murphy tube (ID 3.0-4.0 mm) was inserted through one nostril, after application of topical lidocaine (2\%; Hospira, Lake Forest, IL) to minimize pain and discomfort. When the lambs breathed spontaneously, the endotracheal tube was removed before the nasal tube was attached to a pulsatile Flow Ventilator (VDR4; Percussionaire, Sandpoint, ID) equipped with a humidifier.

Preterm lambs were ventilated for $3 \mathrm{~d}$ or $21 \mathrm{~d}$. Lambs in the IMV group were sedated with pentobarbital sodium (1-3 mg/kg per dose, iv; Abbott Laboratories, North Chicago, IL) as needed to minimize discomfort associated with the endotracheal tube. However, midway into the study, injectable pentobarbital sodium became unavailable. Consequently, the protocol was changed to Fentanyl $(5 \mu \mathrm{g} / \mathrm{kg} /$ dose, Hospira, Lake Forest, IL). Additional sedation was buprenorphine ( $3 \mu \mathrm{g} / \mathrm{kg}$ every $12 \mathrm{~h}$, iv; Buprenex, Reckitt Benckiser Healthcare, Richmond, VA). Lambs in the NIS group were minimally sedated to permit effective spontaneous breathing (pentobarbital sodium, $<1 \mathrm{mg}$ / $\mathrm{kg}$ per dose; Fentanyl $<1 \mu \mathrm{g} / \mathrm{kg} / \mathrm{dose}$ ). All preterm lambs had dextrose and saline solutions administered (iv). Antibiotics were given daily $(100,000 \mathrm{U} / \mathrm{kg}$ potassium penicillin; Pfizer, Groton, CT, and $2.5 \mathrm{mg} / \mathrm{kg}$ gentamicin; Hospira, Lake Forest IL, for the first $10 \mathrm{~d}$ of the $21 \mathrm{~d}$ study). Enteral (orogastric) feeding of sheep colostrum began at $\sim 4 \mathrm{~h}$ of postnatal life. The volume was advanced as tolerated to $60 \mathrm{kcal} / \mathrm{kg} / \mathrm{d}$. For the 21 d groups, orogastric feeding was switched to sheep milk on postnatal day of life 4 and continued for $21 \mathrm{~d}$. The volume of milk was advanced as tolerated. Target for total energy substrate was $150 \mathrm{kcal} / \mathrm{kg} / \mathrm{d}$.

\section{Monitoring}

During respiratory support, we measured heart rate, blood pressure, fractional inspired oxygen $\left(\mathrm{FiO}_{2}\right), \mathrm{PaO}_{2}, \mathrm{PaCO}_{2}, \mathrm{pH}$, peak inspiratory pressure, positive end-expiratory pressure, mean airway pressure, oxygen saturation, hematocrit, plasma sodium, and plasma glucose. Daily measurements included weight, enteral colostrum $/ \mathrm{milk}$ and total (enteral plus intravenous) fluid intake, urine and stool output, rectal temperature, and total and differential white blood cell counts. Because the present assessment of renal development was a secondary analysis, relevant biochemical parameters for renal function (creatinine, Blood urea nitrogen (BUN), glomerular filtration rate) were not measured and could not be measured retrospectively.

\section{Lamb Groups for Normal Developmental Reference}

Fetal lambs were delivered at gestational ages of $130 \mathrm{~d}$ (F130) and 136 $\mathrm{d}$ (F136), bracketing the gestational age at delivery (131-132 d) and age at the end of $3 \mathrm{~d}$ respiratory support (134-135 d), respectively for preterm lambs. None of the ewes for the fetal lambs received prepartum dexamethasone. Fetal lambs were not allowed to breathe before tissue collection. Two groups of term lambs were spontaneously born and left with their mothers for $1 \mathrm{~d}$ after birth (T1d) or $21 \mathrm{~d}$ after birth (T21d). The T1d group provided the gestation age-matched reference for preterm lambs that were supported for $21 \mathrm{~d}$ because preterm lambs were delivered $21 \mathrm{~d}$ early. The T21d group provided the postnatal age-matched reference for the $21 \mathrm{~d}$ preterm lamb groups that were supported for $21 \mathrm{~d}$. The T1d and T21d groups were fed ad libitum by their mothers.

None of the preterm lambs had, or required, urinary bladder catheters. None of the preterm lambs had structural evidence of urinary tract anomalies or obstruction at necropsy.

\section{Postmortem Analyses}

Lambs were given 1,000 U heparin sodium (iv; APP Pharmaceuticals, Schaumberg, IL) followed by pentobarbital sodium $(60 \mathrm{mg} / \mathrm{kg}$; Beuthanasia solution, Ovation Pharmaceuticals, Deerfield, IL). The abdomen was opened and both kidneys were removed. One kidney was cut into uniform slices that were immersed in $10 \%$ neutral buffered formalin at $4^{\circ} \mathrm{C}$ for $24 \mathrm{~h}$. Systematic, uniform, random methods were used for unbiased sampling $(11,18)$ to obtain paraffin-embedded sections ( $5 \mu \mathrm{m}$ thickness).

\section{Immunohistochemistry}

We used a polyclonal rabbit anti-sheep CD-31 antibody (optimal dilution $1: 500)$ made in our laboratory as a capillary endothelial cell marker $(14,19)$. Glomerular capillaries (as well as other capillaries) were uniformly immunostained brown for measurement of $\mathrm{SV}_{\mathrm{sc}}$ lmmunohistochemical staining controls were substitution of the primary antibody with an irrelevant primary antibody (monoclonal mouse antiactin alpha-smooth muscle antibody, Cat. no. A5225, Sigma-Aldrich, St Louis, MO), omission of the primary antibody (replaced with blocking buffer), and omission of the secondary antibody (replaced with blocking buffer).

\section{Quantitative Histologic Analyses}

Glomerular generations and indices of glomerular architecture were measured by computer-assisted image analysis (Bioquant TrueColor Windows, R\&M Biometrics, Nashville, TN). For glomerular generations, five medullary rays were cut longitudinally to count all glomeruli along one side of each medullary ray (20). For glomerular capillarization, we quantified $\mathrm{SV}_{\mathrm{gc}}$. The cortex was visually stratified into three layers of equal depth: inner, middle, and outer cortex. From a randomly chosen starting point, 25 sequential glomeruli each were photographed within the inner and outer stratum at $100 \times$ magnification. A cycloid grid was placed over each digital image. Intersections and endpoints were counted as either crossing immunostained capillaries or lying over tissue, respectively, within a glomerulus. The ratio of counts of capillary intersections over tissue points was calculated for $\mathrm{SV}_{\mathrm{gc}}$ (21). We also measured glomerular surface area (20).

\section{Data analysis}

Results are reported as mean \pm SD. Data were normally distributed (Shapiro-Wilk test); therefore, for physiological results, betweengroup comparison was by unpaired $t$-test, and within-group comparison was by ANOVA followed by Dunnett's post hoc test, using day of life 1 as the comparison age. Likewise for morphometric results, we used ANOVA followed by Fishers PLSD post hoc test. We used 
StatView 5.0 (StatView, Nasbit, MS) and assessed statistical significance at $P<0.05$.

\section{RESULTS}

\section{Demographic Characteristics of Preterm Lamb 3 and $21 \mathrm{~d}$ Groups}

Gestational age at delivery was $\sim 131 \mathrm{~d}$ for the $3 \mathrm{~d}$ groups of preterm lambs (Table 1). Males made up to half of the lamb groups. Birth weight and ending weight were not different among the groups.

For the $21 \mathrm{~d}$ preterm lambs, gestational age at delivery was $\sim 132 \mathrm{~d}$ (Table 2). Males made up one-third of the $21 \mathrm{~d}$ group. Birth weight was not different among groups. Ending weight was not different between the preterm lamb groups, although the change in weight from birth to the end of $21 \mathrm{~d}$ was $0 \mathrm{~kg}$ for the IMV group and $+0.8 \mathrm{~kg}$ for the NIS group. As expected, the T21d reference group weighed more than the preterm lambs.

\section{Physiological Parameters for $\mathbf{3}$ and $\mathbf{2 1}$ d Preterm Lamb groups} For the $3 \mathrm{~d}$ preterm groups (Figure 1), peak inspiratory pressure setting at the ventilator was similar for the IMV and NIS groups. Mean airway pressure setting at the ventilator was significantly higher for the IMV group compared with the NIS group. Positive end-expiratory pressure was kept constant

Table 1. Demographic characteristics of preterm lambs for $3 \mathrm{~d}$ ventilation studies, compared with unventilated fetal reference lambs at matched gestational ages

\begin{tabular}{|c|c|c|c|c|}
\hline & \multirow{2}{*}{$\begin{array}{c}\begin{array}{c}\text { Fetal } \\
\text { reference }\end{array} \\
\text { F130 }\end{array}$} & \multicolumn{2}{|c|}{ Preterm $3 \mathrm{~d}$} & \multirow{2}{*}{$\begin{array}{c}\begin{array}{c}\text { Fetal } \\
\text { reference }\end{array} \\
\text { F136 }\end{array}$} \\
\hline & & IMV & NIS & \\
\hline Sex (Male:Female) & $2: 6$ & $4: 4$ & $3: 5$ & $4: 4$ \\
\hline Gestational age (d) & $131 \pm 1$ & $131 \pm 1$ & $133 \pm 2$ & $136 \pm 1$ \\
\hline Weight at delivery (kg) & $3.1 \pm 0.8$ & $3.7 \pm 0.8$ & $4.8 \pm 1.1$ & $4.0 \pm 1.4$ \\
\hline Weight at study end (kg) & $3.1 \pm 0.8$ & $3.3 \pm 0.9$ & $4.3 \pm 1.1$ & $4.0 \pm 1.4$ \\
\hline
\end{tabular}

(mean \pm SD; $n=8 /$ group). F130 = fetal age 130 d. F136 = fetal age 136 d. Fetal ages of reference lambs match gestational ages of preterm ventilated lambs at beginning and end of the study, respectively,

IMV, invasive mechanical ventilation; NIS, noninvasive support

Table 2. Demographic characteristics of preterm lambs for $21 \mathrm{~d}$ ventilation studies, compared with unventilated fetal and term reference lambs at matched gestational and chronological age

\begin{tabular}{|c|c|c|c|c|c|}
\hline & \multirow{2}{*}{$\begin{array}{c}\begin{array}{c}\text { Fetal } \\
\text { reference }\end{array} \\
\text { F130 }\end{array}$} & \multicolumn{2}{|c|}{ Preterm $21 \mathrm{~d}$} & \multicolumn{2}{|c|}{$\begin{array}{l}\text { Term Reference } \\
\qquad(\sim 150 \mathrm{~d})\end{array}$} \\
\hline & & IMV & NIS & $\mathrm{T}$ & T21d \\
\hline Sex (Male : Female) & $2: 6$ & $2: 6$ & $2: 6$ & $5: 3$ & $5: 3$ \\
\hline Gestational age (d) & $131 \pm 1$ & $132 \pm 2$ & $132 \pm 1$ & & \\
\hline Weight at delivery (kg) & $3.1 \pm 0.8$ & $4.1 \pm 0.8$ & $4.0 \pm 0.8$ & $5.3 \pm 1.7$ & $4.8 \pm 0.4$ \\
\hline Weight at study end (kg) & $3.1 \pm 0.8$ & $4.4 \pm 0.6$ & $4.8 \pm 1.2$ & $5.3 \pm 1.7$ & $14.5 \pm 3.8^{*}$ \\
\hline \multicolumn{6}{|c|}{$\begin{array}{l}\text { (mean } \pm \text { SD; } n=8 / \text { group). } \\
\text { F130 = fetal age } 130 \mathrm{~d} \text {, matching gestational age of preterm ventilated lambs at } \\
\text { beginning of the study. T = unventilated term born lamb on day } 1 \text { of life, matching } \\
\text { gestational age at end of preterm ventilation study. T21d = unventilated term born lamb } \\
\text { on day } 21 \text { of life, matching chronological age at end of preterm ventilation study. } \\
\text { IMV, invasive mechanical ventilation; NIS, noninvasive support; T, term. } \\
{ }^{*} P<0.05 \text { compared with matched preterm ventilated lambs. }\end{array}$} \\
\hline
\end{tabular}

at $8 \mathrm{cmH}_{2} \mathrm{O}$ for both groups (see Supplementary Figure S1 online). $\mathrm{FiO}_{2}$ setting at the ventilator was not different between the two groups and decreased during the first $24 \mathrm{~h}$ of life. These ventilator settings kept arterial blood $\mathrm{pH}, \mathrm{PaCO}_{2}$, and $\mathrm{PaO}_{2}$ within target range (see Supplementary Figure S1 online). Systolic blood pressure and heart rate were not different between the two groups. Total fluid intake (intravenous plus enteral) and enteral milk intake also were not different between the two groups. Urine output, plasma sodium and glucose levels (see Supplementary Figure S1 online) also were not different between the IMV and NIS groups.

We did not measure creatinine, BUN, or glomerular filtration rate because this study was secondary to respiratory gas exchange and alveolarization assessements.

For the $21 \mathrm{~d}$ preterm groups (Figure 2), peak inspiratory pressure and mean airway pressure settings at the ventilator remained constant for the IMV group, whereas peak inspiratory pressure and mean airway pressure settings at the ventilator progressively declined for the NIS group. Positive end-expiratory pressure was kept constant at $8 \mathrm{cmH}_{2} \mathrm{O}$ for both groups (see Supplementary Figure $\mathbf{S} 2$ online). $\mathrm{FiO}_{2}$ setting at the ventilator was significantly lower for the IMV group on the first day of life compared with the NIS group. Thereafter, $\mathrm{FiO}_{2}$ setting at the ventilator was not different between both groups. Arterial blood gas values $\left(\mathrm{pH}, \mathrm{PaO}_{2}\right.$, and $\left.\mathrm{PaCO}_{2}\right)$ were not different and within target range for both groups (see Supplementary Figure S2 online). No differences were detected for systolic blood pressure and heart rate between the two groups. Systolic blood pressure peaked during the second week of life for both groups. Total fluid intake (intravenous plus enteral) and enteral milk intake progressively increased for the NIS group, whereas both intakes decreased for the IMV group. Total fluid intake and enteral milk intake were constant and not different during the first $14 \mathrm{~d}$ of life for both groups. Both intakes decreased significantly during the third week of life for the IMV group, whereas both intakes increased during the same period for the NIS group. Urine output was not different between the two groups, with an exception of lower urine output on day of life 19 for the IMV group $(P<0.05)$. Plasma sodium and glucose levels were not different between the IMV and NIS groups during the $21 \mathrm{~d}$ study period (see Supplementary Figure S2 online).

\section{Indices of Glomerular Number and Maturation for $\mathbf{3}$ and $\mathbf{2 1} \mathrm{d}$ Preterm Lamb Groups}

Preterm lambs that were supported by IMV or NIS for $3 \mathrm{~d}$ did not have differences in the number of glomerular generations (Figure 3). We saw no histological evidence of immature or shrunken glomeruli in either the inner or outer cortex. Immunostaining of glomerular capillaries is shown in Figure 4. We used the immunostained sections for $\mathrm{SV}_{\mathrm{gc}}$ measurements. $\mathrm{SV}_{\mathrm{gc}}$ and glomerular surface area were not different between the IMV and NIS groups, regardless of cortical region.

Figure 3 includes results for normal development for contrast with the results for the preterm lamb groups. Glomerular generations were not different among the F130 and F136 
a

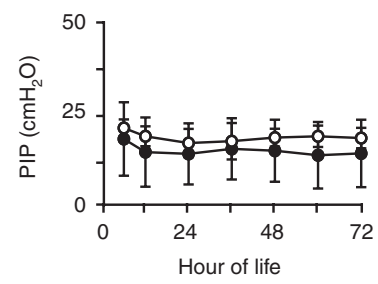

e

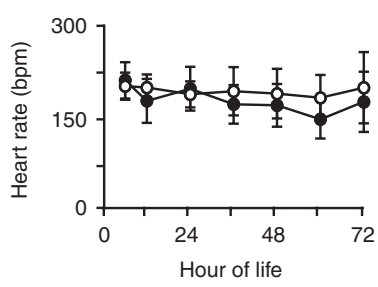

b

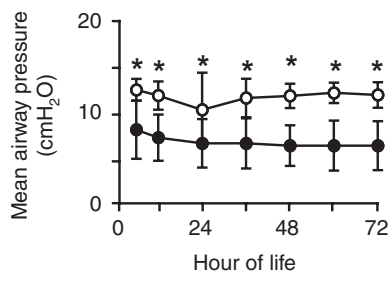

f

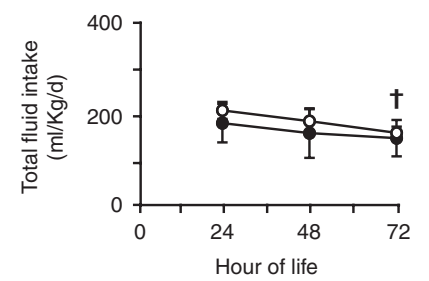

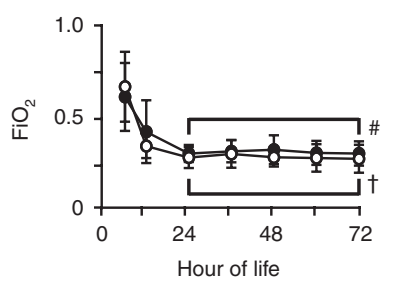

g

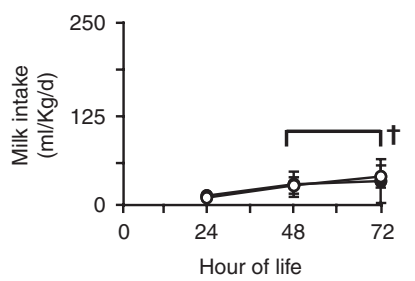

d

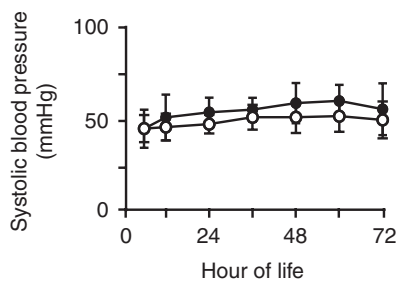

h

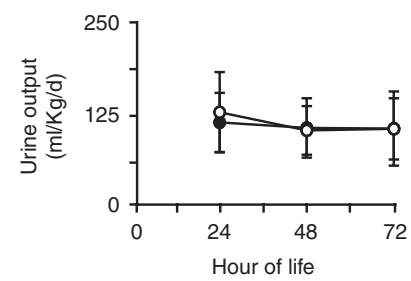

Figure 1. Physiological parameters for $3 \mathrm{~d}$ preterm lamb groups. Parameters measured at $12 \mathrm{~h}$ intervals for (a) peak inspiratory pressure (PIP), (b) mean airway pressure, (c) fractional inspired oxygen $\left(\mathrm{FiO}_{2}\right)$, (d) systolic blood pressure, (e) heart rate, (f) total fluid intake (intravenous plus enteral), (g) enteral milk intake, and (h) urine output for preterm lambs that were supported by invasive mechanical ventilation (IMV; white circles) or noninvasive support (NIS; black circles) over the first $72 \mathrm{~h}$ of life. Between-groups comparisons at indicated hour-of-life by unpaired $t$-test ${ }^{*} P<0.05$ compared with NIS group, and within-group by ANOVA and Dunnett's post-hoc test $\left({ }^{\dagger} P<0.05\right.$ compared with $\mathrm{d} 1 \mathrm{IMV} ;{ }^{*} P<0.05$ compared with d1 NIS).

a

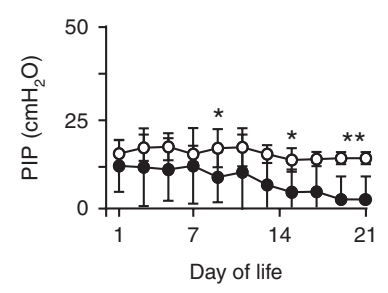

e

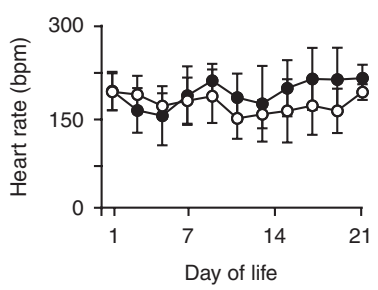

b

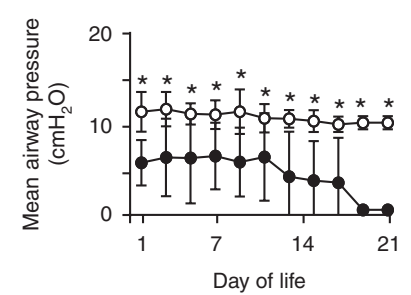

f

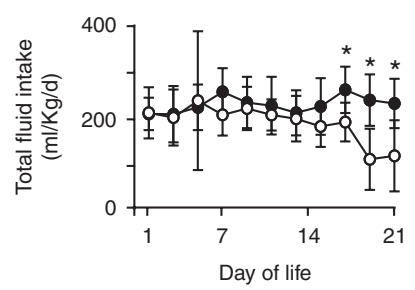

C

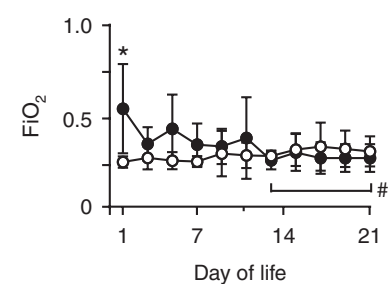

g

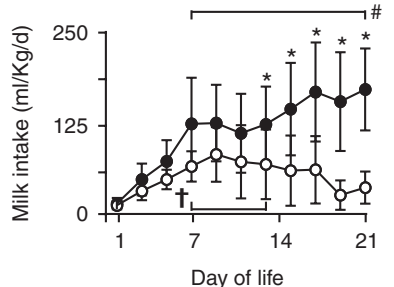

d

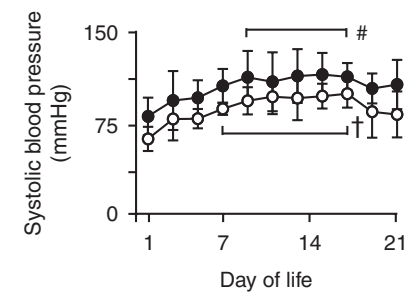

h

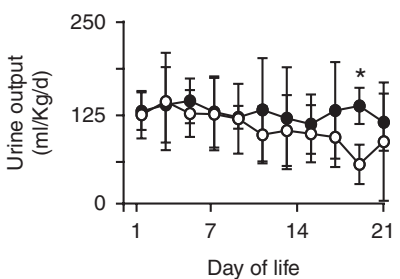

Figure 2. Physiological parameters for $21 \mathrm{~d}$ preterm lamb groups. Parameters measured at $24 \mathrm{~h}$ intervals for (a) peak inspiratory pressure (PIP), (b) mean airway pressure, (c) fractional inspired oxygen $\left(\mathrm{FiO}_{2}\right)$, (d) systolic blood pressure, (e) heart rate, (f) total fluid intake (intravenous plus enteral), (g) enteral milk intake, and (h) urine output for preterm lambs that were supported by invasive mechanical ventilation (IMV; white circles) or noninvasive support (NIS; black circles) over $21 \mathrm{~d}$ of life. Between-groups comparisons at indicated day-of-life by unpaired $t$-test $\left({ }^{*} P<0.05\right.$ compared with NIS group), and within-group by ANOVA and Dunnett's post-hoc test $\left({ }^{+} P<0.05\right.$ compared with d1 IMV; ${ }^{P} P<0.05$ compared with d1 NIS).

reference groups and preterm lamb groups. $\mathrm{SV}_{\mathrm{gc}}$ was significantly greater for glomeruli located in the inner cortex of the F130 reference group compared with both preterm lamb groups. $\mathrm{SV}_{\mathrm{gc}}$ was significantly greater for the outer cortex of the F130 and F136 reference groups compared with both preterm lamb groups. Glomerular surface area was significantly less for glomeruli located in the inner cortex of the F130 reference group compared with preterm lambs supported by IMV and NIS. Glomerular surface area was significantly less for the outer cortex of the F130 and F136 reference groups compared with the NIS preterm lamb group.
Preterm lambs that were supported by IMV or NIS for $21 \mathrm{~d}$ also did not have differences in the number of glomerular generations (Figures 4 and 5). $\mathrm{SV}_{\mathrm{gc}}$ was not different between the IMV and NIS groups, regardless of cortical region. Glomerular surface area was significantly less for glomeruli in the inner cortex of the IMV group compared with the NIS group. For the outer cortex, glomerular surface density was not different between the IMV and NIS groups.

The panels in Figure 5 likewise provide contrasting results for normal development. Glomerular generations were not different among the reference groups and preterm lamb groups. 
$\mathrm{SV}_{\mathrm{gc}}$ was significantly greater for glomeruli located in the inner cortex among the F130, T1d, and T21d groups compared with preterm lambs supported by IMV or NIS. Glomerular surface area was significantly less for glomeruli located in the inner cortex within the F130 reference group compared with the NIS group of preterm lambs. The $21 \mathrm{~d}$ reference group had significantly greater glomerular surface area than the NIS group of preterm lambs. Glomerular surface area for the outer cortex was significantly less for the F130 reference group compared with both NIS preterm lamb group. The $21 \mathrm{~d}$ reference group had significantly greater glomerular surface area than the NIS group of preterm lambs.

\section{DISCUSSION}

The principal finding of our study is that moderate preterm birth and $3 \mathrm{~d}$ or $21 \mathrm{~d}$ of respiratory support, either IMV or NIS, impairs postnatal development of $\mathrm{SV}_{\mathrm{gc}}$ and glomerular surface area. Glomerular generations were not affected, as expected because the preterm lambs were delivered after glomerulogenesis is completed in sheep. Contrary to our hypothesis, early initiation of NIS after premature birth does not minimize the glomerular impairments compared with IMV. Thus, we conclude that moderate preterm birth and respiratory support, whether invasive or noninvasive, impairs glomerular architecture in preterm lambs. This adverse effect on glomerular maturation may contribute to increased risk for adult-onset hypertension and renal dysfunction.

The rationale for this study is based on comparison of the mode of respiratory support on lung outcomes for preterm lambs $(14,15)$. Briefly, noninvasive respiratory support of preterm lambs improves respiratory gas exchange and alveolarisation in the lung compared with IMV. Better physiologi$\mathrm{cal}$ and morphological outcomes for the lung during NIS are also evident in the brain of preterm lambs (16) and baboons (22). Those findings led to this secondary analysis of the kidneys. The detrimental effect of prematurity accompanied by IMV specifically on glomerular architecture development after completion of glomerulogenesis in this study is consistent with reduced total renal filtration surface and reduced glomerular capillary length during IMV in moderately preterm lambs (23). Our study adds the insight that NIS also decreases glomerular architectural development equally in the kidney of moderately premature lambs.

Abnormally shaped glomeruli, fewer glomerular generations, and glomerular hypertrophy are abnormal structural findings in autopsy studies of preterm human infants who died with BPD and necropsy studies of mechanically ventilated preterm baboons $(10,11)$. Our study did not detect abnormally shaped glomeruli or fewer glomerular generations. The species-specific relation between gestational age at delivery and the gestational age at which glomerulogenesis is complete may account for the differences between studies. Glomerulogenesis in humans is completed by $\sim 36 \mathrm{wk}$ gestation $(1,10,24)$, so preterm birth earlier makes glomerulogenesis vulnerable to disruption. Likewise, glomerulogenesis in baboons finishes by $\sim 175 \mathrm{~d}$ gestation, whereas the preterm baboon model uses

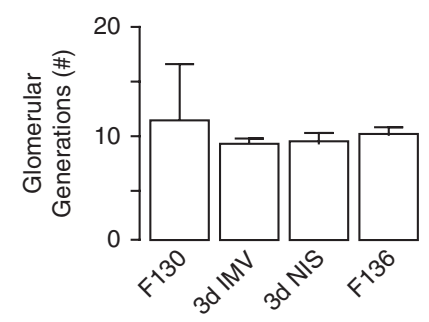

b
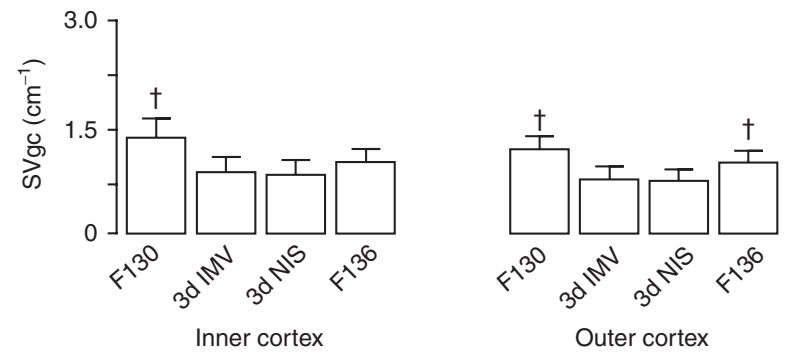

C
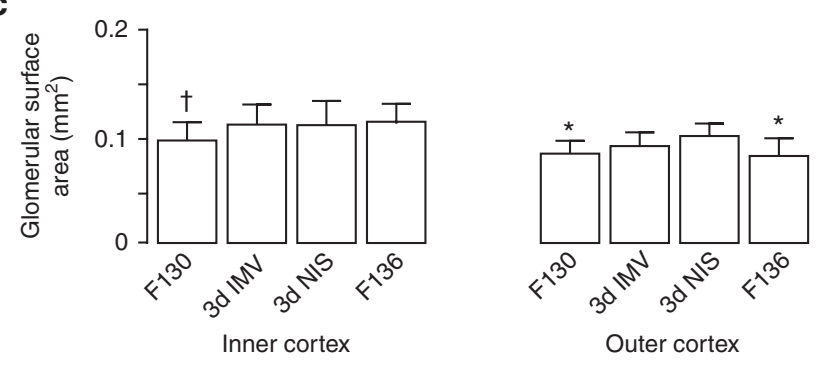

Figure 3. Morphometric results for glomerular architecture for $3 \mathrm{~d}$ preterm lamb groups. Glomerular generations (a), glomerular capillary surface density (b), and glomerular surface area (c) were not different between the invasive mechanical ventilation (IMV) and noninvasive support (NIS) groups. For normal developmental reference, unventilated, untreated fetal (F) lambs bracket the delivery age in days (F130) and ending age (F136) of the preterm lambs. Between-groups comparisons by ANOVA and Fishers PLSD post-hoc test $\left({ }^{+} P<0.05\right.$ compared with IMV and NIS; ${ }^{*} P<0.05$ compared with NIS).

delivery $\sim 50 \mathrm{~d}$ earlier (25). We deliver preterm lambs $\sim 10 \mathrm{~d}$ after glomerulogenesis is completed in sheep $(\sim 120 \mathrm{~d}$ gestation) (26). Lambs delivered at gestational age earlier than 120 $\mathrm{d}$ are unlikely to survive despite respiratory and other support, based on our experience (27).

Our study shows that $\mathrm{SV}_{\mathrm{gc}}$ is greater in the kidney of normal term lambs that are 3 wk old compared with fetal lambs and term lambs that are $1 \mathrm{~d}$ old (Figure 5b). Postnatal growth of glomerular capillaries may reflect the rise in functional demand after birth. Vascular resistance falls and renal blood flow increases postnatally with concomitantly greater glomerular filtration rate in the first $24 \mathrm{~h}$ of postnatal life in sheep (28) and further increase in glomerular filtration rate during the first weeks and months of postnatal life in lambs (29) and humans (30). The increased perfusion of the kidneys may trigger lengthening and branching of glomerular capillaries, reflected in increased filtration area. Further studies will be necessary to determine the timecourse of normal postnatal glomerular capillary lengthening and branching. 

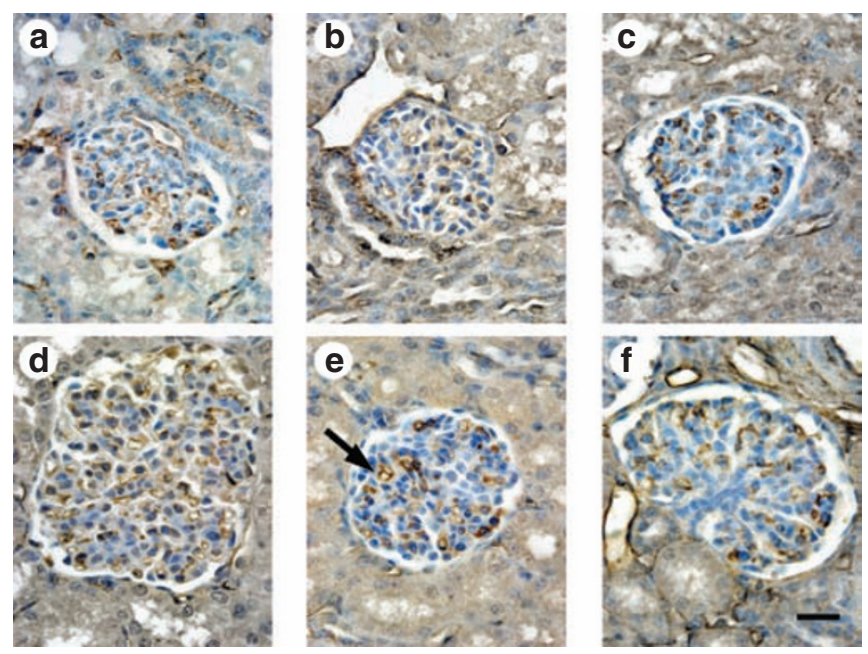

Figure 4. Immunohistochemical localization of CD-31 to identify glomerular capillaries. Anti-CD-31 antibody provided uniform immumolocalization of glomerular capillary endothelial cells (brown color; black arrow in e). Images are representative of the morphometric results shown in Figures 3 and 5. (a) Unventilated, untreated fetal lamb at $136 \mathrm{~d}$ gestation (F136), (b) preterm lamb at 3 d IMV, (c) preterm lamb at 3 d NIS, (d) unventilated, untreated term $21 \mathrm{~d}$-old lamb (T21d), (e) preterm lamb at $21 \mathrm{~d}$ IMV, (f) preterm lamb at $21 \mathrm{~d}$ NIS. The scale bar is 20 micrometers in length.

IMV may alter kidney function in adults by reducing renal blood flow. We did not measure renal blood flow, owing to the secondary nature of our study, so it does not provide mechanistic insight. Interestingly, the $21 \mathrm{~d}$ IMV group of preterm lambs had lower systolic blood pressure (not statistically significant) than the NIS group, which might reduce renal blood flow. Another caveat is that blood pressure is not necessarily proportional to blood flow in preterm subjects (31).

Our study was not designed to test independent effects of other factors that may influence glomerular architecture the preterm period, either fetal or postnatal. These include exposure to antenatal steroids, respiratory support with oxygenrich gas, systemic hypotension, exposure to aminoglycosides, sedatives, intrauterine and extrauterine growth restriction, and fluid and dietary intakes. Antenatal steroid exposure prior to the completion of glomerulogenesis in sheep ( 120 d gestation) decreases nephron endowment $(32,33)$. Whether antenatal steroid exposure after $120 \mathrm{~d}$ gestation in sheep affects glomerular architectural maturation remains to be determined. Oxygen-exposure of preterm neonates, including the preterm lambs reported here, was higher postnatally than in utero and therefore could inhibit vascular endothelial growth factor expression, leading to reduced glomerular capillary development $(34,35)$. Systemic hypotension did not occur in the preterm lambs. Also, systolic blood pressure did not differ between the IMV and NIS groups. Our study design minimized potential nephrotoxicity among the $21 \mathrm{~d}$ ventilated groups of preterm lambs by stopping Gentamicin at $10 \mathrm{~d}$ of treatment, as is done clinically. All of the preterm lambs, regardless of mode of ventilation, were exposed to antenatal steroid and postnatal aminoglycoside antibiotic, and thus were treated uniformly. a

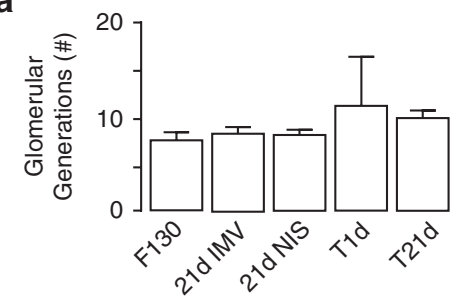

b
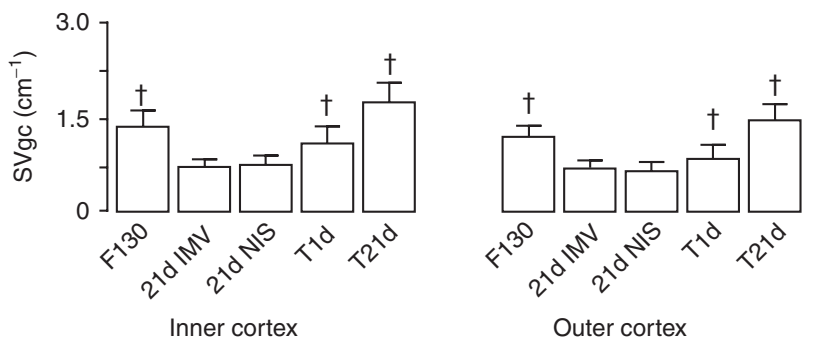

C

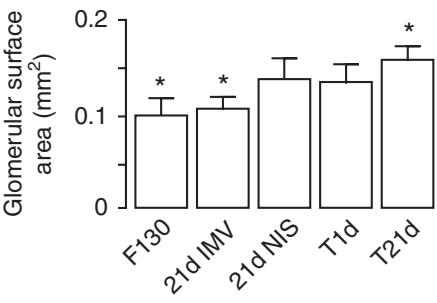

Inner cortex

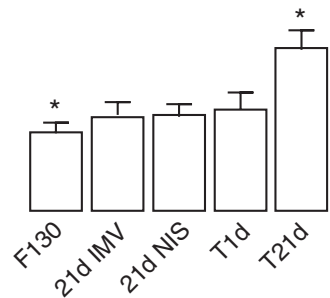

Outer Cortex
Figure 5. Morphometric results for glomerulogenesis and glomerular architecture for $21 \mathrm{~d}$ preterm lambs. Glomerular generations (a), glomerular capillary surface density (b), and glomerular surface area (c) were not different between the invasive mechanical ventilation (IMV) and noninvasive support (NIS) groups. For normal developmental reference, unventilated, untreated fetal lambs match gestational age at delivery (F130) and unventilated, untreated term lambs match gestational age that preterm lambs would have been if not delivered prematurely (T1d) and postnatal age of the preterm lambs (T21d). Data assessed between-groups by ANOVA and Fishers PLSD post-hoc test $\left(^{+} P<0.05\right.$ compared with IMV and NIS; ${ }^{*} P<0.05$ compared with NIS).

This uniform design emulates the neonatal intensive care setting for the majority of preterm human infants.

A potential confounding element of study design is the IMV group which received more sedative (pentobarbital)compared with the NIS group (36) to prevent discomfort or pain from the endotracheal tube, and to minimize breathing over the ventilator. Larger amounts of sedative may decrease blood pressure, renal blood flow, and renal vascular regulation $(37,38)$, which could affect glomerular architecture. However, glomerular architecture was altered the same in the IMV and NIS groups. Thus, differences in the amount of sedative between IMV and NIS did not affect glomerular architectural.

Total fluid intake (IV fluids plus enteral milk) and enteral milk intake alone were lower in the IMV group of preterm lambs only in the last week of the $21 \mathrm{~d}$ study compared with the NIS group. This difference was due to feeding intolerance of the IMV group, evident as residual milk in the stomach. Because enteral milk intake diminished over the last $7 \mathrm{~d}$, IV fluid administration was increased to sustain total fluid intake 
(targeted at $\sim 160 \mathrm{ml} / \mathrm{kg} / \mathrm{d}$ ). Interestingly, despite greater total intake of fluids for the NIS group during the same period, $\mathrm{SV}_{\mathrm{gc}}$ was not different compared with the IMV group. Therefore, total fluid intake or enteral milk intake does not appear to influence glomerular architecture in preterm lambs at the end of $21 \mathrm{~d}$ of respiratory support. This contrasts with findings in human adults that show larger intravenous volume abrogates hemodynamic effects on the kidney $(8,9)$.

Our study design included untreated, unventilated fetal lambs, and term lambs for normal developmental reference from preterm birth at $\sim 131 \mathrm{~d}$ gestation to term-plus-21 d postnatal age. To provide this context, none of the reference lambs were exposed to antenatal steroid or postnatal aminoglycoside antibiotic. Consequently, our study did not assess the independent effect of either treatment on normal glomerular architecture.

Our study has methodological limitations because of its secondary design. Measurements of kidney weight or volume were not assessed during the original studies. Also, we used morphometric methods, rather than stereological methods (39), to quantify glomerular architecture. We also used paraffin-embedded tissue blocks for the morphometric analyses for economy of time and cost for preparing tissue blocks from multiple organs from the same lambs. Nonetheless, we used random, systematic, and uniform sampling approaches and a cycloid grid $(21,40)$. We used the same approaches for all lamb groups, so any effects of analytical approach are common among the groups. Nonetheless, our findings of significantly reduced $\mathrm{SV}_{\mathrm{gc}}$ represent an important starting point for future stereological assessments of glomerular architecture in ventilated preterm lambs.

We also did not measure renal function, such as creatinine, BUN, or glomerular filtration rate. This limitation is the consequence of secondary assessment after analysis of lung alveolarisation $(14,15)$. Without measures of renal function, uncertainty remains about evidence for renal dysfunction in the preterm lamb groups. Indirectly, Figures $\mathbf{1}$ and $\mathbf{2}$ show that urine output was normal (typically $1-3 \mathrm{ml} / \mathrm{kg} / \mathrm{h}$ ) and equivalent between the IMV and NIS groups, as were plasma sodium and glucose levels (in supplemental material). We currently measure serum creatinine and BUN, and urinary microalbumin, with attention to the postnatal adaptive changes of renal function in newborn and preterm ventilated subjects (25-27).

We consider prematurity and respiratory support (IMV and NIS) to be two influential factors on glomerular architecture. A new report by our group focused on prematurity as an influential factor by comparing glomerular stereological parameters between preterm and term lambs (23). That study provides the insight that regardless of being preterm or term, lambs supported by IMV for $3 \mathrm{~d}$ have significantly decreased glomerular capillary length and surface area, and reduced total renal filtration surface area. This study provides a different insight; namely, that the mode of respiratory support, either IMV or NIS for $3 \mathrm{~d}$ or $21 \mathrm{~d}$ of preterm lambs does not influence glomerular architecture in the kidney. Together, the two studies are complementary and suggest that the immature kidney is vulnerable to prolonged respiratory support. Mechanisms remain to be identified.

In summary, moderate preterm birth after completion of glomerulogenesis and prolonged respiratory support (invasive or noninvasive) impair glomerular architectural development. These adverse effects during the early period of postnatal life in preterm neonates may increase risk for systemic hypertension and renal dysfunction later in life.

\section{SUPPLEMENTARY MATERIAL}

Supplementary material is linked to the online version of the paper at http:// www.nature.com/pr

\section{ACKNOWLEDGMENTS}

We thank Angela Presson, Division of Epidemiology, University of Utah and Lukas Staub, Institute of Social and Preventive Medicine, University of Bern, Switzerland, for statistical guidance.

\section{STATEMENT OF FINANCIAL SUPPORT}

This study was supported by National Institutes of Health $(\mathrm{NIH})$ grants HL110002 and HL062875 (KHA), the Division of Neonatology, and the University of Utah Undergraduate Research Opportunities Program. E.S. was supported by a Fellowship from the Gottfried and Julia Bangerter-Rhyner Foundation, Basel (Switzerland).

Disclosure: The authors have no financial ties to products used in this study.

\section{REFERENCES}

1. Hinchliffe SA, Sargent PH, Howard CV, Chan YF, van Velzen D. Human intrauterine renal growth expressed in absolute number of glomeruli assessed by the disector method and Cavalieri principle. Lab Invest 1991;64:777-84.

2. Rodríguez MM, Gómez AH, Abitbol CL, Chandar JJ, Duara S, Zilleruelo GE. Histomorphometric analysis of postnatal glomerulogenesis in extremely preterm infants. Pediatr Dev Pathol 2004;7:17-25.

3. Hughson M, Farris AB 3rd, Douglas-Denton R, Hoy WE, Bertram JF. Glomerular number and size in autopsy kidneys: the relationship to birth weight. Kidney Int 2003;63:2113-22.

4. Bacchetta J, Harambat J, Dubourg L, et al. Both extrauterine and intrauterine growth restriction impair renal function in children born very preterm. Kidney Int 2009;76:445-52.

5. Duncan AF, Heyne RJ, Morgan JS, Ahmad N, Rosenfeld CR. Elevated systolic blood pressure in preterm very-low-birth-weight infants $\leq 3$ years of life. Pediatr Nephrol 2011;26:1115-21.

6. Frankfurt JA, Duncan AF, Heyne RJ, Rosenfeld CR. Renal function and systolic blood pressure in very-low-birth-weight infants 1-3 years of age. Pediatr Nephrol 2012;27:2285-91.

7. Van Marter LJ, Allred EN, Pagano M, et al. Do clinical markers of barotrauma and oxygen toxicity explain interhospital variation in rates of chronic lung disease? The Neonatology Committee for the Developmental Network. Pediatrics 2000;105:1194-201.

8. Koyner JL, Murray PT. Mechanical ventilation and the kidney. Blood Purif 2010;29:52-68.

9. Pannu N, Mehta RL. Effect of mechanical ventilation on the kidney. Best Pract Res Clin Anaesthesiol 2004;18:189-203.

10. Sutherland MR, Gubhaju L, Moore L, et al. Accelerated maturation and abnormal morphology in the preterm neonatal kidney. J Am Soc Nephrol 2011;22:1365-74.

11. Gubhaju L, Sutherland MR, Yoder BA, Zulli A, Bertram JF, Black MJ. Is nephrogenesis affected by preterm birth? Studies in a non-human primate model. Am J Physiol Renal Physiol 2009;297:F1668-77.

12. Saxel L. Organogenesis of the Kidney. Cambrige, CA: Cambrige University Press; 1987.

13. Schmölzer GM, Kumar M, Pichler G, Aziz K, O’Reilly M, Cheung PY. Non-invasive versus invasive respiratory support in preterm infants at birth: systematic review and meta-analysis. BMJ 2013;347:f5980. 


\section{Articles | staubetal.}

14. Reyburn B, Li M, Metcalfe DB, et al. Nasal ventilation alters mesenchymal cell turnover and improves alveolarization in preterm lambs. Am J Respir Crit Care Med 2008;178:407-18.

15. Null DM, Alvord J, Leavitt W, et al. High-frequency nasal ventilation for 21 $\mathrm{d}$ maintains gas exchange with lower respiratory pressures and promotes alveolarization in preterm lambs. Pediatr Res 2014;75:507-16.

16. Albertine $\mathrm{KH}$. Brain injury in chronically ventilated preterm neonates: collateral damage related to ventilation strategy. Clin Perinatol 2012;39: $727-40$.

17. Gimonet V, Bussieres L, Medjebeur AA, Gasser B, Lelongt B, Laborde K. Nephrogenesis and angiotensin II receptor subtypes gene expression in the fetal lamb. Am J Physiol 1998;274(6 Pt 2):F1062-9.

18. Sutherland MR, Gubhaju L, Black MJ. Stereological assessment of renal development in a baboon model of preterm birth. Am J Nephrol 2011;33 Suppl 1:25-33.

19. Albertine KH, Dahl MJ, Gonzales LW, et al. Chronic lung disease in preterm lambs: effect of daily vitamin A treatment on alveolarization. Am J Physiol Lung Cell Mol Physiol 2010;299:L59-72.

20. Sutherland MR, Gubhaju L, Yoder BA, Stahlman MT, Black MJ. The effects of postnatal retinoic acid administration on nephron endowment in the preterm baboon kidney. Pediatr Res 2009;65:397-402.

21. Nyengaard JR. Stereologic methods and their application in kidney research. J Am Soc Nephrol 1999;10:1100-23.

22. Loeliger M, Inder T, Cain S, et al. Cerebral outcomes in a preterm baboon model of early versus delayed nasal continuous positive airway pressure. Pediatrics 2006;118:1640-53.

23. Sutherland MR, Ryan D, Dahl MJ, Albertine KH, Black MJ. Effects of preterm birth and ventilation on glomerular capillary growth in the neonatal lamb kidney. J Hypertens 2016;34:1988-97.

24. Takano K, Kawasaki Y, Imaizumi T, et al. Development of glomerular endothelial cells, podocytes and mesangial cells in the human fetus and infant. Tohoku J Exp Med 2007;212:81-90.

25. Gubhaju L, Black MJ. The baboon as a good model for studies of human kidney development. Pediatr Res 2005;58:505-9.

26. Gimonet V, Bussieres L, Medjebeur AA, Gasser B, Lelongt B, Laborde K. Nephrogenesis and angiotensin II receptor subtypes gene expression in the fetal lamb. Am J Physiol 1998;274(6 Pt 2):F1062-9.

27. Albertine $\mathrm{KH}$, Jones GP, Starcher BC, et al. Chronic lung injury in preterm lambs. Disordered respiratory tract development. Am J Respir Crit Care Med 1999;159:945-58.
28. Nakamura KT, Matherne GP, McWeeny OJ, Smith BA, Robillard JE. Renal hemodynamics and functional changes during the transition from fetal to newborn life in sheep. Pediatr Res 1987;21:229-34.

29. Aperia A, Broberger O, Herin P. Maturational changes in glomerular perfusion rate and glomerular filtration rate in lambs. Pediatr Res 1974;8:75865 .

30. Saint-Faust M, Boubred F, Simeoni U. Renal development and neonatal adaptation. Am J Perinatol 2014;31:773-80.

31. Evans N. Assessment and support of the preterm circulation. Early Hum Dev 2006;82:803-10

32. Figueroa JP, Rose JC, Massmann GA, Zhang J, Acuña G. Alterations in fetal kidney development and elevations in arterial blood pressure in young adult sheep after clinical doses of antenatal glucocorticoids. Pediatr Res 2005;58:510-5.

33. Moritz KM, De Matteo R, Dodic M, et al. Prenatal glucocorticoid exposure in the sheep alters renal development in utero: implications for adult renal function and blood pressure control. Am J Physiol Regul Integr Comp Physiol 2011;301:R500-9.

34. Sutherland MR, Bertagnolli M, Lukaszewski MA, et al. Preterm birth and hypertension risk: the oxidative stress paradigm. Hypertension 2014;63:12-8.

35. Tufro-McReddie A, Norwood VF, Aylor KW, Botkin SJ, Carey RM, Gomez RA. Oxygen regulates vascular endothelial growth factor-mediated vasculogenesis and tubulogenesis. Dev Biol 1997;183:139-49.

36. Joss-Moore LA, Hagen-Lillevik SJ, Yost C, et al. Alveolar formation is dysregulated by restricted nutrition but not excess sedation in preterm lambs supported by non-invasive ventilation. Ped Res 2016;80(5):719-728.

37. Linas SL, Berl T, Aisenbrey GA, Better OS, Anderson RJ. The effect of anesthesia on hemodynamics and renal function in the rat. Pflugers Arch 1980;384:135-41.

38. Priano LL. Effects of high-dose fentanyl on renal haemodynamics in conscious dogs. Can Anaesth Soc J 1983;30:10-8.

39. Guo M, Ricardo SD, Deane JA, Shi M, Cullen-McEwen L, Bertram JF. A stereological study of the renal glomerular vasculature in the $\mathrm{db} / \mathrm{db}$ mouse model of diabetic nephropathy. J Anat 2005;207:813-21.

40. Hsia CC, Hyde DM, Ochs M, Weibel ER; ATS/ERS Joint Task Force on Quantitative Assessment of Lung Structure. An official research policy statement of the American Thoracic Society/European Respiratory Society: standards for quantitative assessment of lung structure. Am J Respir Crit Care Med 2010;181:394-418. 